\title{
Chelating properties of the calixarene carboxylic
}

\author{
Pascal Chanton ${ }^{1}$
}

Laboratoire CEVIDRA, France

IRSN demonstrated that a nanoemulsion loaded with calixarene carboxylic is much more efficient for the decontamination of skin contaminated by actinides than the existing products $[1,2,3,4]$. This innovation has been patented by IRSN and an exploitation license has been granted to a Pharmaceutical Laboratory

The efficacy of the IRSN product is based on the known complexation activity of the calixarene carboxylic on actinides, and on integrating this chelating agent in a nanoemulsion.

A cleansing nanoemulsion formulation has been developed by IRSN on the advice of the physicians of the nuclear industry. This cleansing formulation of calixarene carboxylic demonstrated a similar efficacy to the patented nanoemulsion [5].

This cleansing nanoemulsion has been developed to be used as an emergency treatment at the time of actinides contamination or potential contamination, the product acting by chelating the soluble elements of actinides and by cleansing insoluble elements present on the skin/hairs.

An industrial cleansing nanoemulsion has been made and marketed in compliance with the IRSN one. It was therefore important to better determine the chelating properties of the calixarene carboxylic when loaded in the industrial cleansing nanoemulsion to better define the scope of use of this marketed product.

\section{Summary}

The Calixarene carboxylic is a the p-tert-butylcalix[6]arene bearing 3 carboxylic groups arranged in $\mathrm{C} 3$ symmetry with a cone conformation allowing the formation of a pseudo planar hexacoordinate complex with Uranium, displaying a high affinity (6).

This property was initially used by IRSN for the analysis of contaminated urine / water. The use of calixarene columns (LCarbH3 and LHydH3) allowed a quantitative and reproducible separation of Uranium, Plutonium and Americium from mineralized urine samples (7). Thorium extraction from mineralised urine samples on calixarene columns was feasible and demonstrated with quantitative yields (8).

The chelating properties of the calixarene carboxylic in a nano emulsion was assessed first by performing the "In vitro" study developed by IRSN. In a second step, IRSN demonstrated that specific chelating capacities of the nano emulsion allowed an efficient decontamination of skin using an "Ex-vivo" study model.

The "In vitro" study performed by IRSN against Uranium, Cobalt, Caesium and Strontium shows that the Calixarene CEVIDRA's cleansing nano emulsion exhibits good

\footnotetext{
${ }^{1}$ Corresponding author: p.chanton@cevidra.com
} 
affinity towards actinides such as Uranium, but also towards other elements such as Cobalt, Strontium or Caesium.

Compared to previous studies, a relatively lower extraction efficacy regarding Uranium can be observed. This would be potentially due to a one step extraction of 4 mixed elements instead of one element extraction in previous studies. This one step extraction of 4 mixed elements could also induce competition between Uranium and the other "lighter" and more diffusible elements

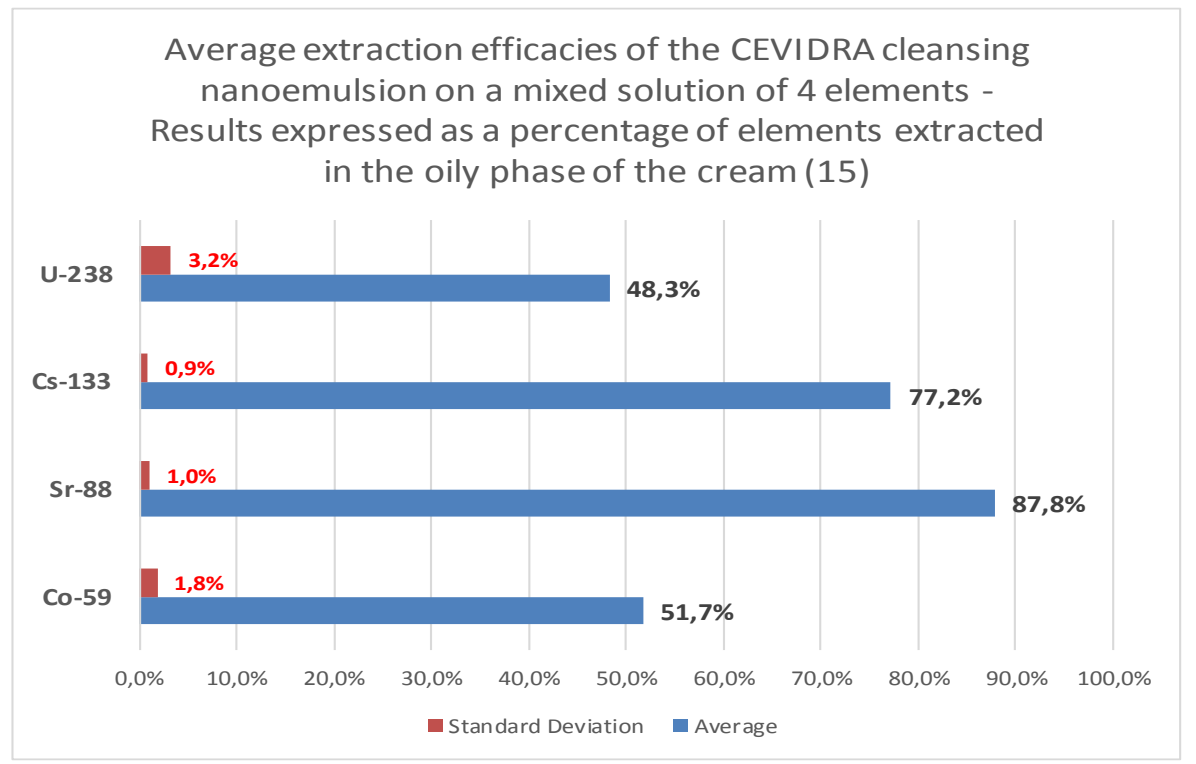

These results allow CEVIDRA to extend the use of the product to the treatment of Cobalt, Caesium and/or Strontium skin and hair contaminations.

The safety studies performed on the active ingredient and performed on the CEVIDRA's cleansing nano emulsion, demonstrated the good tolerance and the very low toxicity of this product.

Recently, the use of the CEVIDRA nano emulsion on real cases of skin and hair contaminations confirmed the high decontamination efficacy of the product.

These elements reinforce the importance of using the CEVIDRA nano emulsion for the emergency treatment of contamination or suspicion of contamination to Actinides (Uranium, Plutonium, Americium and Thorium), Cobalt, Caesium and Strontium.

\section{References}

1. Spagnul, A., Bouvier-Capely, C., Phan, G., Rebière, F. et Fattal, E. (2010). "Calixarene-entrapped nanoemulsion for uranium extraction from contaminated solutions". Journal of Pharmaceutical Sciences, 99(3), 1375-1383

2. Spagnul, A., Bouvier-Capely, C., Adam, M., Phan, G., Rebière, F. et Fattal, E. (2010). "Quick and efficient extraction of uranium from a contaminated solution by a calixarene nanoemulsion". International Journal of Pharmaceutics, 398(1-2), 179-184

3. Spagnul, A., Bouvier-Capely, C., Phan, G., Landon, G., Tessier, C., Suhard, D., Rebière, F., Agarande, M. et Fattal, E. (2011). "Ex vivo decrease in uranium diffusion 
through intact and excoriated pig ear skin by a calixarene nanoemulsion". European Journal of Pharmaceutics and Biopharmaceutics, 79(2), 258-267

4. Grives, S., Phan, G., Morat, G., Suhard, D., Rebiere, F. et Fattal, E. (2015). "Ex vivo uranium decontamination efficiency on wounded skin and in vitro skin toxicity of a calixarene-loaded nanoemulsion". Journal of Pharmaceutical Sciences, 104(6), 20082017

5. Phan, G., Semili, N., Bouvier-Capely, C., Landon, G., Mekhloufi, G., Huang, N., Rebière, F., Agarande, M. et Fattal, E. (2013). "Calixarene cleansing formulation for uranium skin contamination". Health Physics, 105(4), 382-389

6. Fattal E, Tsapis N, Phan G. Novel drug delivery systems for actinides (uranium and plutonium) decontamination agents. Adv Drug Deliv Rev. 2015 Aug 1;90:40-54.

7. Bouvier-Capely, C., Manoury, A., Legrand, A., Bonthonneau, J. P., Cuenot, F. et Rebière, F. (2009). "The use of calix[6]arene molecules for actinides analysis in urine and drinking water: An alternative to current procedures". Journal of Radioanalytical and Nuclear Chemistry, 282(2), 611-615

8. Mekki, S., Bouvier-Capely, C., Jalouali, R. et Rebière, F. (2010). " The extraction of thorium by calix[6]arene columns for urine analysis ". Radiation Protection Dosimetry, 144(1-4), 330-334 